\title{
Downregulation of IncRNA-HOXA11-AS modulates proliferation and stemness in Glioma cells
}

\author{
Zuoxin Zhang ${ }^{1,2,3}$, Junhu Zhou ${ }^{1,2,3}$, Junxia Zhang ${ }^{4}$, Ran Duan ${ }^{5}$, Peiyu Pu ${ }^{1,2,3}$ and Lei Han ${ }^{1,2,3^{*}}$
}

\begin{abstract}
Background: Glioma stem cells (GSCs) represent a subpopulation of cells within glioma that are characterized by chemotherapy resistance and tumor recurrence. GSCs are therefore important therapeutic target for glioma therapy. Long non-coding RNAs (IncRNAs) have been shown to regulate important functions in cancer. HOXA11-AS is one such IncRNA and has been shown to regulate cell proliferation via promotion of cell cycle progression in glioblastoma (GBM) cells. However, the specific roles of HOXA11-AS in GSCs remain unclear.
\end{abstract}

Methods: Here we investigated the role of HOXA11-AS in driving GSC stemness properties via sphere-forming and protein chip assays.

Results: Gain-of-function as well as loss-of-function results showed that the HOXA11-AS maybe a critical modulator in GBM recurrence as demonstrated by cell sphere-forming ability. Furthermore, we showed that induced expression of HOXA11-AS does increase the levels of stemness-related transcription factors (Oct4/Sox17/Sox2) in U87MG cells. In vivo xenograft experiments using the HOXA11-AS knockdown U87MG cells revealed that downregulation of HOXA11-AS could strongly inhibit tumor growth. Furthermore, we found that HOXA11-AS knockdown decreased the expression of cancer stemness markers in vivo.

Conclusions: Collectively, these data suggests that HOXA11-AS is involved in GSC stemness and supports its clinical significance as a important therapeutic target in glioma.

Keywords: Glioma, LncRNA, HOXA11-as, Glioma stem cells

\section{Background}

Glioblastoma (GBM) is the highest grade glioma (grade IV) in adults and remains incurable despite promising treatments for its heterogeneous features [1]. These phenotypes are thought primarily to be due to their derivation from glioma stem cells (GSCs), which are a highly tumorigenic and capable of self-renewal subpopulation of glioma cells [2]. As the self-renewing capability and multipotency may be closely associated with cancer progression and invasion, understanding the mechanisms underlying the regulation of stemness properties of glioma cells has been widely investigated.

\footnotetext{
* Correspondence: superhanlei@hotmail.com

${ }^{1}$ Department of Neurosurgery, Tianjin Medical University General Hospital,

154\#, Anshan Road, Heping District, Tianjin 300052, China

${ }^{2}$ Lab of Neuro-oncology, Tianjin Neurological Institute, Tianjin, China

Full list of author information is available at the end of the article
}

Long non-coding RNAs (long ncRNAs, lncRNA) are a class of non-protein coding transcripts with a length longer than 200 nucleotides. Noncoding RNAs (ncRNA) may be transcribed from noncoding DNA, which represents over $98 \%$ of human genome DNA and is commonly refered to as "junk DNA" [3]. To date, studies have shown that IncRNAs affect the chromatin structure, mRNA stability and miRNA-mediated gene regulation by acting as competing endogenous RNA or natural miRNA sponges [4]. HOXA11 antisense (HOXA11-AS) was in 5-prime region of the HOXA locus. To date, only a few investigations have reported a association between HOXA11-AS and cancer. Richards et al. showed that HOXA11-AS could inhibit the proliferation and invasion of ovarian cancer via a series of functional assays [5]. Sun $M$ et al. found that the $5^{\prime}$ region of HOXA11-AS binds to chromatin-modifying complexes (PRC2 and 
LSD1), hence inhibiting the transcription of its target genes (PRSS8 and KLF2) [6]. Our group has demonstrated that HOXA11-AS knockdown inhibits the cell cycle progression and proliferation of glioma cells [7]; however, the specific roles of HOXA11-AS in pluripotency regulation are still largely unknown.

Here, to explore the underlying molecular mechanisms of HOXA11-AS in the proliferation and stemness properties of GBM, we investigate the biological functions of HOXA11-AS in regulating the proliferation and maintenance of stemness by overexpression and knockdown of HOXA11-AS in U87MG cells in vitro and in vivo. Our observations show that HOXA11-AS is an effective modulator that regulates the glioma stem cell population.

\section{Methods}

\section{Patients and samples}

mRNA expression profile for glioma and normal tissues were got from the Chinese Glioma Genome Atlas (CGGA, http://cgga.org.cn/) [8]. Microarray analysis in 220 glioma cases (97 grade II, 34 grade III, and 89 GBM) and 5 normal brain samples was performed via the Agilent Array.

\section{Cell culture and viral infection}

Human GBM cell lines U87MG, LN229, U251 and human embryonic kidney 293 (HEK293) cells were purchased from ATCC (the American Type Culture Collection, Manassas, VA, USA). The U87-EGFRvIII cell line, which carries EGFR vIII mutation, was kindly provided by Prof. Ren of Harbin Medical University (Harbin, China) [9]. All GBM cell lines were routinely cultured at $37{ }^{\circ} \mathrm{C}$ in a $5 \% \mathrm{CO}_{2}$ humidified atmosphere in Dulbecco's-modified Eagle medium (DMEM) with 10\% fetal bovine serum (FBS, HyClone). Lentivirus containing HOXA11-AS RNAi segments (the RNAi sequence is $5^{\prime}$ GCAGCAUGCUUGUGCUCAATT-3') and the luciferase reporter gene were obtained from Genepharma (Shanghai, China). Dox-inducible HOXA11-AS expression recombinant adenovirus was purchased from SinoGenoMax (Beijing, China). Doxycycline (dox) was purchased from Santa Cruz (Dallas, TX, USA). The GBM cell lines were infected with virus suspensions for $48 \mathrm{~h}$ according to the manufacturer's instructions.

\section{Quantitative real-time PCR}

Total RNA from glioma cells was extracted using TRIzol reagent (Life Technologies). The GoScript ${ }^{\mathrm{Tm}}$ Reverse Transcription System (Promega, USA) can be used to synthesize the single stranded $\mathrm{CDNA}$ via reverse transcribing total RNA. To identify HOXA11-AS and GAPDH mRNA expression level, quantitative reverse transcription-polymerase chain reaction (qRT-PCR) was performed using a CFX96 Real-Time PCR Detection
System (Bio-Rad Laboratories). The sequence of $\mathrm{HO}$ XA11-AS primer is as follows: Sense: $5^{\prime}$-CCCTCAT CTTCCCTGCCTACCT-3', antisense: 3'-GGTTTCCC GAGTCCTCAGCT-5'.

\section{Sphere formation assay}

The sphere-forming assays were performed [10]. Briefly, cells $\left(1 \times 10^{3} /\right.$ well $)$ were cultured in ultra low attachment 6-well plates in $2 \mathrm{~mL}$ of stem cell culture media and the media was supplemented with $2 \%$ B27, $20 \mathrm{ng} / \mathrm{mL}$ EGF and $20 \mathrm{ng} / \mathrm{mL}$ FGF-b on every other day. After 10 days, the cell spheres with a size larger than $50 \mu \mathrm{m}$ were photographed and counted per well via microscope.

\section{Analysis of stem cell marker by protein microarray}

After infected by virus for $48 \mathrm{~h}$, the U87MG cells were washed with PBS buffer and lysed with RIPA solution (Pierce, France) with the protease/phosphatase inhibitor cocktails (Sigma, USA). The expression of the human stem cell markers were analyzed by RayBio Human Stem Cell Array G1, none of the spots was under detectable fluorescence level and, therefore, we can calculate significance for all markers.

\section{Nude mouse glioma intracranial model}

Female $\mathrm{Nu} / \mathrm{Nu}$ nude mice (aged 4-5 weeks) were purchased from the animal center at the cancer institute, Chinese Academy of Medical Science (Beijing, China). We infected U87MG cells with HOXA11-AS RNAi and luciferase lentivirus for $48 \mathrm{~h}$. Then, we implanted stereotactically a total of $2.0 \times 10^{5}$ U87MG glioma cells into the brain of mice to establish intracranial xenografts model [11]. Each group has 13 mice. The fluorescent imaging of tumor progression in the orthotopic mouse model was monitored via the IVIS Lumina Imaging System (Xenogen, Waltham, MA, USA) every 7 days for three times. After 20 days of treatment, three mice per group were sacrificed and brains were collected and embedded in paraffin for immunohistochemical (IHC) staining. The remaining ten mice in each group were used for survival analysis.

\section{Immunohistochemical analysis}

For hematoxylin and eosin (HE) staining, the sections were placed in the distilled water before nucleus is stained with hematoxylin. Then, the sections were stained with eosin before being dehydrated and mounted. For IHC staining, the paraffin-embedded tissue sections were used for the examination the expression of Oct-4, and Sox-2. Sections were dewaxed, treated with $\mathrm{H}_{2} \mathrm{O}_{2}$, and then incubated with the primary antibodies (1:100; Santa Cruz Biotechnology) overnight at $4{ }^{\circ} \mathrm{C}$, followed by incubation with biotinylated secondary antibody (1:100; Santa Cruz Biotechnology) for $1 \mathrm{~h}$ at room temperature. After 
washing with PBS, the sections were incubated with 3,3'-diaminobenzidine (DAB) for approximately $1 \mathrm{~min}$, and counterstained with hematoxylin.

\section{Statistical analyses}

The one-way analysis of variance (ANOVA), and $t$-test were used to determine statistically significant difference between different groups. Kaplan-Meier method analysis was used to assess the survival distributions, and the log-rank test was used to analyze the statistical significance. The cut-off point between high and low values for HOXA11-AS was its median values. Heat maps were generated via TreeView and clustering software. All data are presented as the mean \pm standard error.

\section{Results}

Identification of HOXA11-AS in the mRNA microarray dataset

Since ncRNAs play a crucial role in cancer progression, we wanted to get the expression profile of ncRNAs in the CGGA database. Firstly, we combined the Agilent028002 microarray dataset (1736 ncRNA probes), the lncRNA dataset (182 annotated lncRNAs) and the functional lncRNA databset (172 annotated lncRNAs) to obtain 1997 annotated noncoding RNAs. Secondly, we can further obtain 732 annotated ncRNAs within the CGGA dataset via overlapping the aboved 1997 ncRNAs with the 24,000 probes of the CGGA dataset (Fig. 1a). Thirdly, we further obtained the differential expressed ncRNAs (SAM: Fold change $>1.5$, Q value $<1 \%$ ) among the glioma samples in different grade via SAM analysis. Lastly, we obtained the expression profile of several tumorigenesis-related ncRNAs, which revealed the increased or reduced expression upon glioma grade. Among these ncRNAs, we focus on a novel lncRNA HOXA11-AS for its unknown function in glioma (Fig. 1b).

\section{HOXA11-AS regulates stemness qualities of GBM cell}

To further investigate the effect of HOXA11-AS on stemness characteristics, the expression level of HOXA11-AS in HEK293, U87MG, U87vIII, U251 and LN229 cell lines was firstly measured via qPCR. The expression level of HOXA11-AS in HEK293 cells was used as a control. The results showed that the expression level of HOXA11-AS in U87MG and U87vIII cells was lower than that in LN229 and U251 cells (Fig. 2a). It has been demonstrated that EGFRvIII could promote self-renewal and tumor progression of glioma stem cells [12]. However,we found that
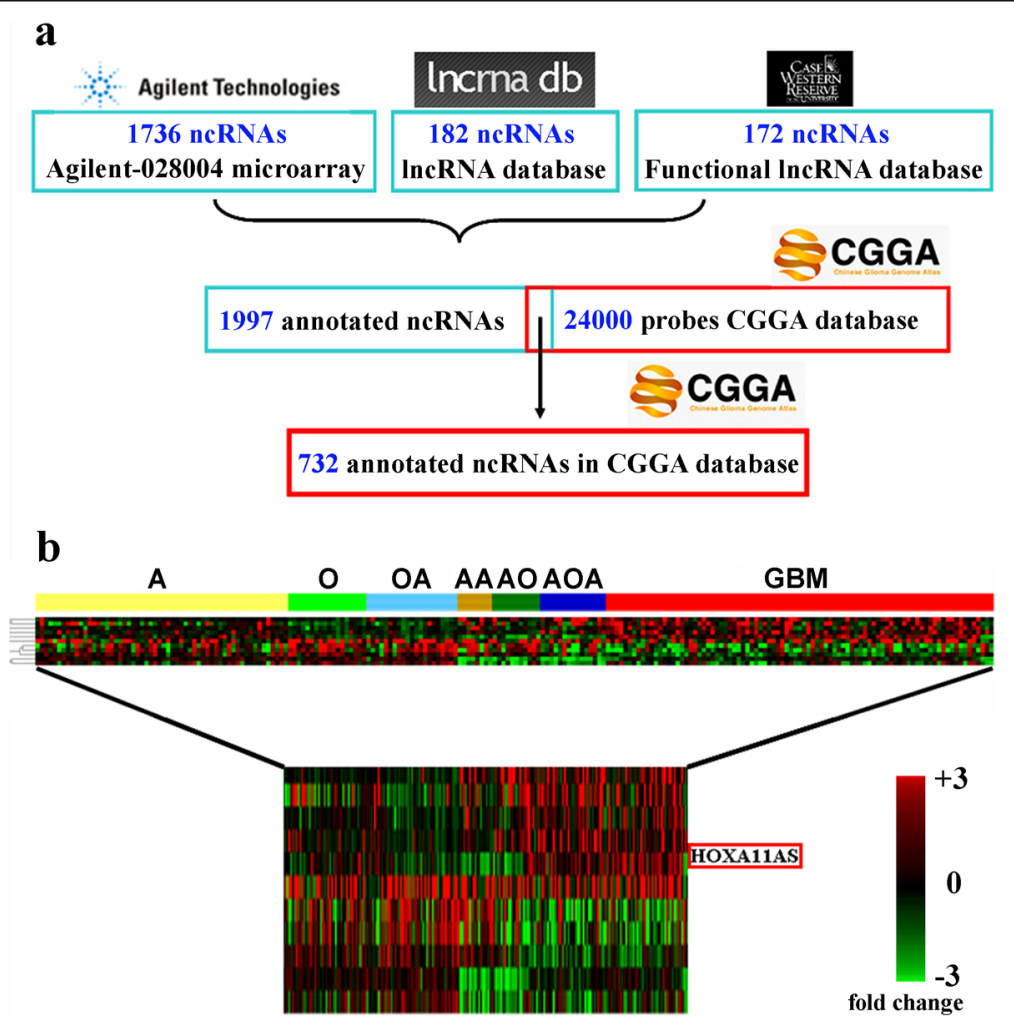

Fig. 1 NcRNA expression signature in the CGGA database and evidence that the novel IncRNA- HOXA11-AS confers poor prognosis in glioma patients. a Non-coding RNAs were obtained from CGGA, the IncRNA database and the functional IncRNA database. $\mathbf{b}$ Sum analysis of the correlation of glioma grade with its associated IncRNAs in CGGA database 


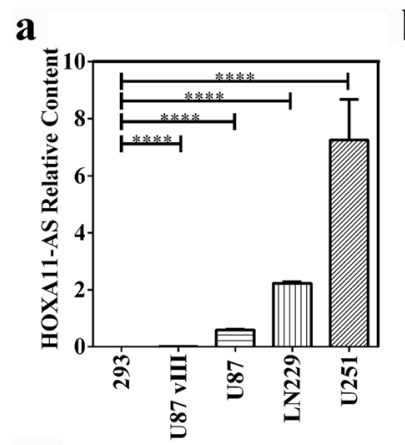

d

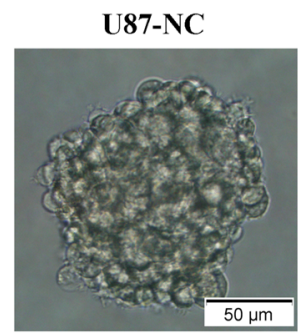

$\mathbf{e}$

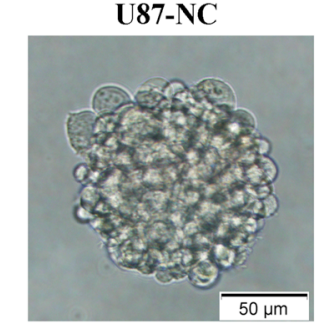

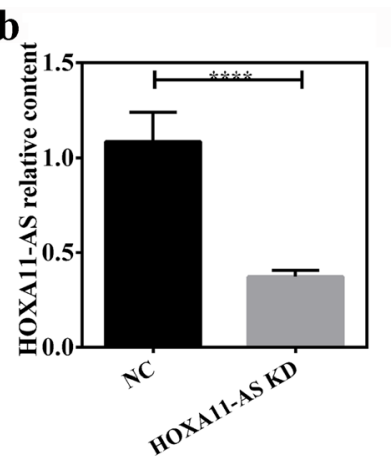

C
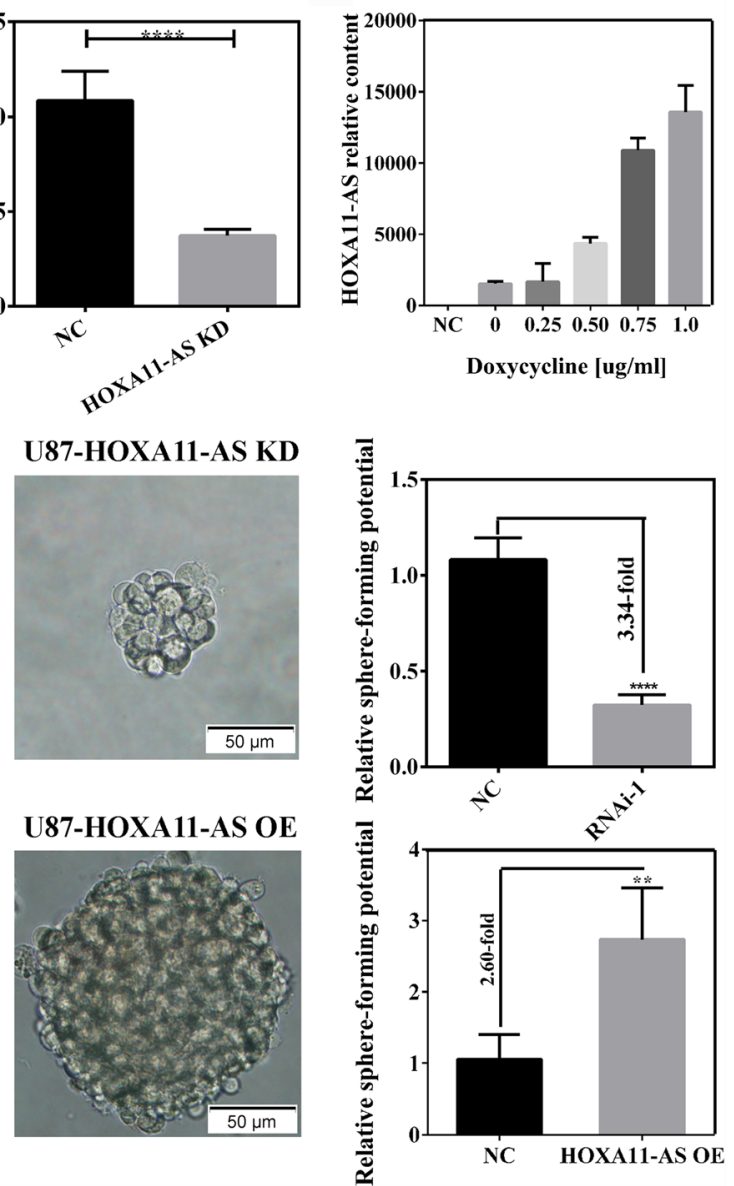

Fig. 2 HOXA11-AS regulates glioma stemness in vitro. a mRNA expression of HOXA11-AS genes was determined in GBM cell lines and normal epithelial cell cultures by qRT-PCR. Expression levels are shown relative to the expression in HEK293 cells, which was used as a control and set as 1. $\mathbf{b}$ qRT-PCR was used to detect HOXA11-AS expression of U87MG cells with lenti-HOXA11-AS knockdown. c qPCR panels represent HOXA11-AS expression in U87MG cells infected with lentivirus-HOXA11-AS overexpression and incubated with different concentrations of doxycyclin (dox) in the culture medium for 2 days. $\mathbf{d}$ and $\mathbf{e}$ HOXA11-AS expressing or silenced U87MG cells $\left(1 \times 10^{3}\right)$ were seeded in ultra-low attachment plate and allowed to grow for 10 days in stem cell culture medium. Images were taken under light microscope and the number of spheres (size $>50 \mu m$ ) was counted. $\left({ }^{* *} p<0.01 ;{ }^{* * *} p<0.0001\right)$

the expression of HOXA11-AS was the lowest in U87vIII cell lines among the four GBM cell lines, which maybe due to the EGFR antagonism in U87vIII cell lines [13].

To address whether HOXA11-AS could regulate stemness characteristics in glioma cells, HOXA11-AS were overexpressed or knockdown in U87MG cells via adenovirus or lentivirus infection, respectively. It was shown that HOXA11-AS RNAi virus could significantly decrease the expression of HOXA11-AS in U87MG cells compared with control (Fig. 2a), while rAd-HOXA11-AS virus could also significantly increase the expression of HOXA11-AS in U87MG cells compared with the control (Fig. 2c). Next, we characterized the stemness qualities of the U87MG cells in vitro via the sphere-forming assay. HOXA11-AS knockdown could impair its sphere-forming ability (Fig. 2d), while HOXA11-AS overexpression could promote the sphere formation of U87MG cells than that in control group (Fig. 2e).

\section{HOXA11-AS has a marked effect on the stemness of GBM cells}

To determine molecular stemness mechanisms of HOXA11-AS, we detected associations between the expression levels of HOXA11-AS and stem cell markers in CGGA cohort, suggesting that HOXA11-AS expression was positively related to expression of BMX, CD15, CD133, EZH2, GATA4, nestin, and PDX1. Furthermore, we further performed protein chips using protein lysates obtained from U87MG cell lines treated with HOXA11AS overexpression adenovirus, or control adenovirus for 
$48 \mathrm{~h}$. We found that the HOXA11-AS overexpression in U87MG cells resulted in increasing stem cell markers expression (Oct-4, Sox2, and Sox17 et al.) (Fig. 3b and c).

\section{HOXA11-AS promotes glioma progression in vivo}

To investigate the effect of HOXA11-AS on proliferation and stemness in vivo, the U87MG cell lines with knockdown of HOXA11-AS were orthotopically injected into brain of nude mice. To monitor treatment responses in vivo, U87MG cells were infected with lentivirus to stably express firefly luciferase prior to intracranial implantation. It was shown that HOXA11-AS knockdown could resist the progression of U87MG cells in vivo (Fig. 4a and b). The survival time of mice in HOXA11-AS knockdown group was prolonged compared with that in control group (Fig. 4c). HE staining of xenograft brain samples further demonstrated that the ratio of tumor growth resisted in the HOXA11-AS knockdown group compared with that of the control group. The stemness marker (Oct-4, Sox-2) were obviously lower in HOXA11-AS knockdown group compared with that in control group (Fig. 4d). In a word, these results showed that HOXA11-AS could enhance glioma proliferation and stemness in vivo.

\section{Discussion}

GBM is the most common and deadliest of malignant primary brain tumor. Even after current standard treatment with successful surgical resection, combination of radiotherapy with chemotherapy (temozolomide), GBM are associated with a poor prognosis due to chemotherapy resistance and histopathological heterogeneity [14]. Gliomas contain relatively undifferentiated cells called GSCs, which are associated with gliomagenesis, therapeutic resistance, and inevitable recurrence [15]. Therefore, to explore the underlying molecular mechanisms of stemness properties of GSCs would have great clinical implications for better treatment of glioma. In this research, for the first time, we investigated the effect of HOXA11-AS on the stemness characteristics in glioma via detecting the expression of stemness-relateded markers, and sphere-forming ability.

Enhanced sphere-forming ability and resistance to treatment (chemotherapy and radiotherapy) are the two common properties of CSCs. As we all know, the treatment resistance is mainly attributed to self-renewing capacity of CSCs in the minority subpopulation of cancer cells. Previous studies have demonstrated that GSCs enhance the chemo-resistance of glioma to TMZ. In this work, in vivo as well as in vitro results showed that HOXA11-AS knockdown not only decreased the expression of stemness markers in protein level, but also dramatically impaired the sphere-forming ability of GBM cells. We also noticed the enhanced expression of stemness-related markers (including Oct4, Sox2, Sox17, and Nanog) in HOXA11-AS overexpressed GBM cells

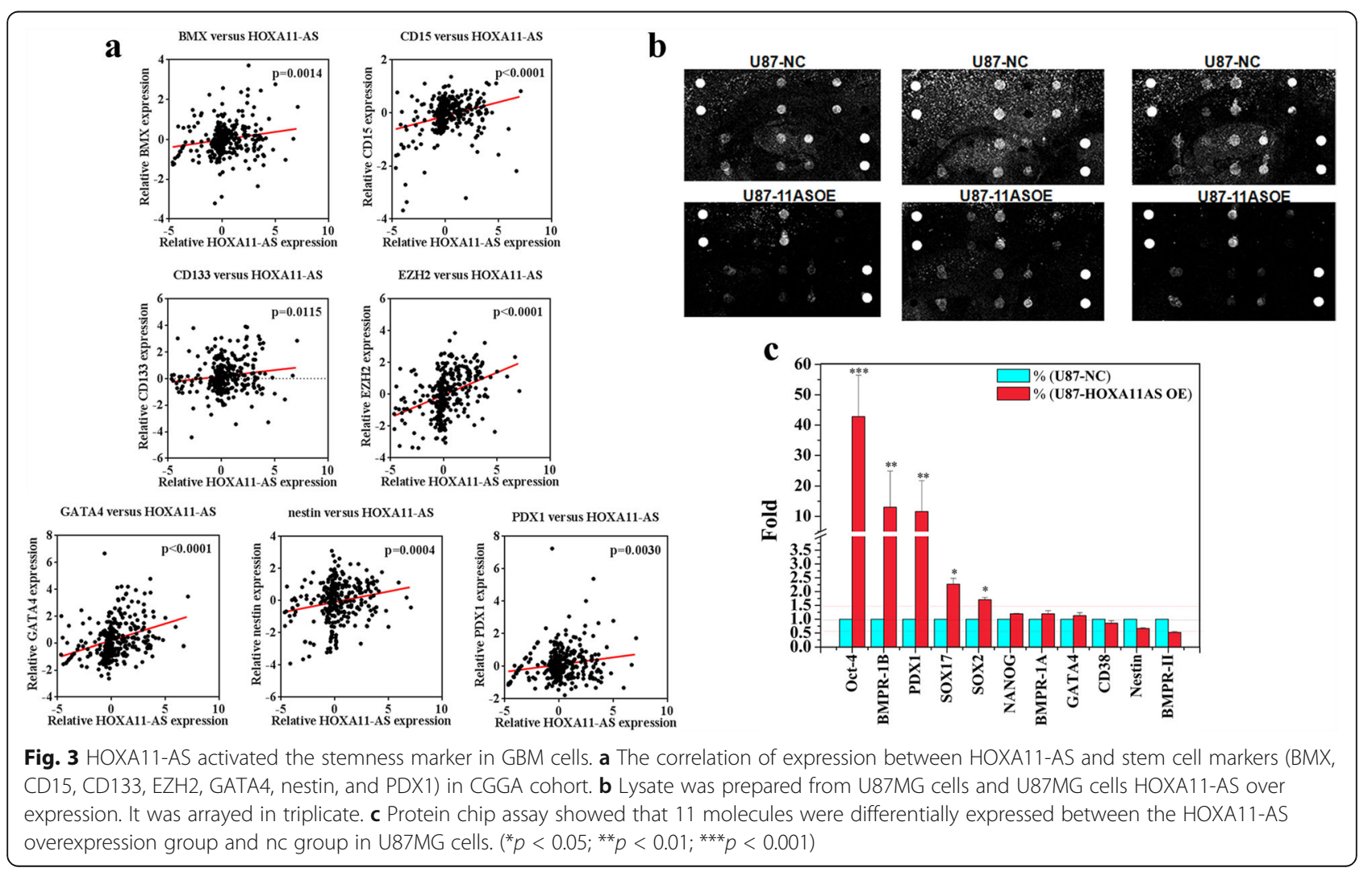



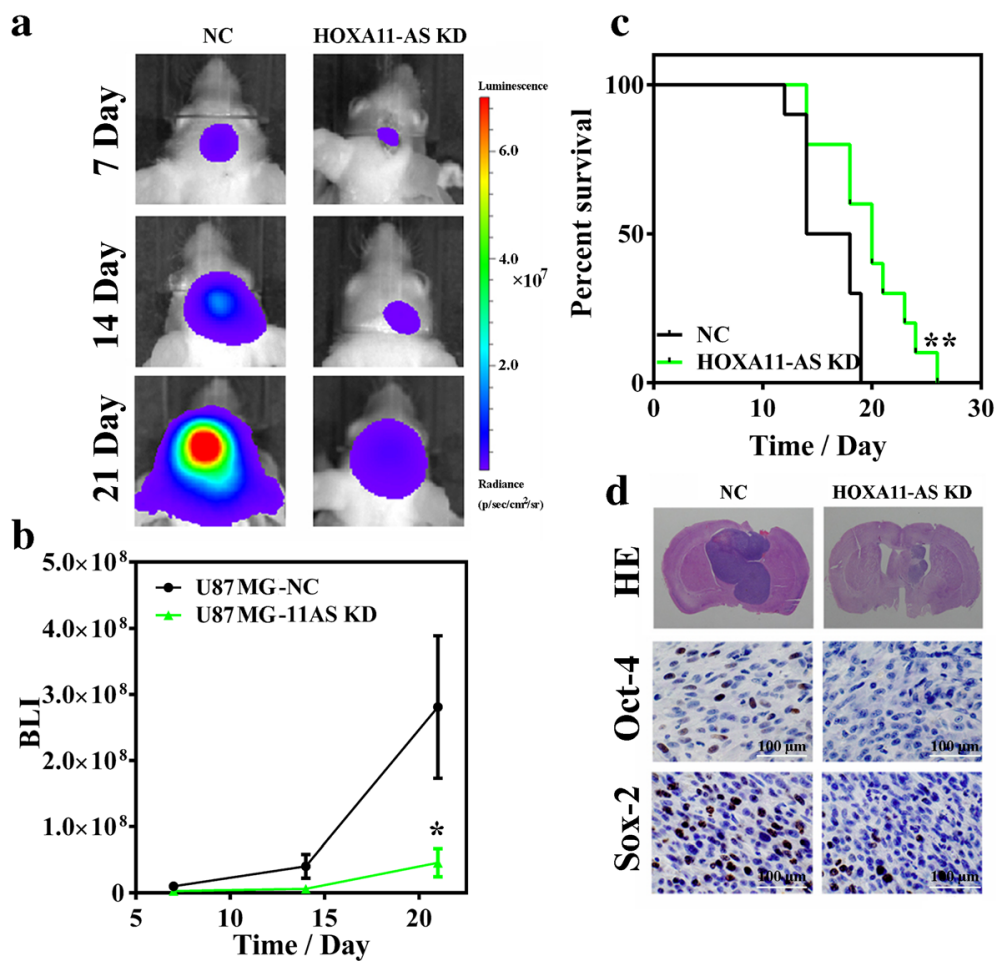

Fig. 4 HOXA11-AS expression associated with tumor growth rate and stemness markers in an intracranial glioma murine xenograft model (a) U87MG cells pretreated with a lentivirus with HOXA11 RNAi, or NC and lentivirus containing luciferase were implanted into the brains of nude mice, and tumor formation was assessed by bioluminescence imaging. Changes in bioluminescent signal were detected at days 7, 14, and 21 day after implantation. b Bioluminescence (BLI) was monitored to assess the tumor growth at days 7, 14, and 21. c Overall survival was determined by Kaplan-Meier survival curves and log-rank test was used to assess the statistical significance of the differences. $\mathbf{d}$ Tissue slices were stained with Hematoxylin-eosin-saffron. Representative images of immunohistochemical staining for Oct-4, and Sox-2. $\left({ }^{*} p<0.05 ;{ }^{* *} p<0.01\right)$

via protein microarray. Previous studies have demonstrated that the Oct4, Sox2, Sox17, and Nanog are the important regulator of stemness in the different types of cancer including GBM [16, 17]. In summary, our findings further indicate that HOXA11-AS maybe involve in maintenance of stemness in glioma.

\section{Conclusions}

This study demonstrates that lncRNAs HOXA11-AS are involved in regulating the stemness of glioma cells. As discussed above, HOXA11-AS could be significant for not only targeting therapy to the bulk glioma cells, but also affecting the stemness of glioma. Therefore, selective inhibition of HOXA11-AS activity in glioma should provide new opportunities for the glioma therapy.

\section{Abbreviations}

GBM: Glioblastoma; GSCs: Glioma stem cells; HOXA11-AS: HOXA11 antisense; IncRNAs: Long non-coding RNAs

\section{Funding}

Supported by the grant (No. 2014AA021102) from National High Technology Research and Development Program of China 863, the grant (No. 2016YFC0902502) from the National Key Research and Development Plan, the grant (Nos. 81,572,496, 51,473,119, 81,001,128) from National Nature Science Foundation of China. The funding organizations had no role in development of the study or the drafting of the manuscript for publication.

\section{Availability of data and materials}

All the data we needed was presented in the tables and figures in the main paper.

\section{Authors' contributions}

Conception and design: LH; Development of methodology: ZZ, JZ, RD; Data analysis: JZ, LH; Writing, check, and revision of the manuscript: ZZ, LH; Study supervision: PP. All authors read and approved the final manuscript.

Ethics approval and consent to participate

Approval No. TMUaMEC 2,016,004 of Animal Ethicaland by the Animal Ethical and Welfare Committee (AEWC).

Consent for publication

Not applicable.

Competing interests

The authors declare that they have no competing interest. 


\section{Author details}

'Department of Neurosurgery, Tianjin Medical University General Hospital, 154\#, Anshan Road, Heping District, Tianjin 300052, China. ${ }^{2}$ Lab of Neuro-oncology, Tianjin Neurological Institute, Tianjin, China. ${ }^{3}$ Key Laboratory of Post-Neuroinjury Neuro-repair and Regeneration in Central Nervous System, Ministry of Education and Tianjin City, Tianjin 300052, China.

${ }^{4}$ Department of Neurosurgery, The First Affiliated Hospital of Nanjing Medical University, Nanjing 210029, China. ${ }^{5}$ Department of Neurosurgery, Beijing Tian Tan Hospital, Capital Medical University, Beijing, China.

Received: 29 December 2016 Accepted: 10 July 2017

Published online: 13 September 2017

\section{References}

1. Guelfi S, Duffau H, Bauchet L, Rothhut B, Hugnot JP. Vascular Transdifferentiation in the CNS: a focus on neural and Glioblastoma stemlike cells. Stem Cells Int. 2016;2016:2759403.

2. Liebelt BD, Shingu T, Zhou X, Ren J, Shin SA, Hu J. Glioma stem cells: signaling, microenvironment, and therapy. Stem Cells Int. 2016;2016: 7849890 .

3. Quinn JJ, Chang HY. Unique features of long non-coding RNA biogenesis and function. Nat Rev Genet. 2016;1:47-62.

4. Schmitt AM, Chang HY. Long noncoding RNAs in cancer pathways. Cancer Cell. 2016:4:452-63.

5. Richards EJ, Permuth-Wey J, Li Y, Chen YA, Coppola D, Reid BM, Lin HY, Teer JK, Berchuck A, Birrer MJ, Lawrenson K, Monteiro AN, Schildkraut JM, Goode EL, Gayther SA, Sellers TA, Cheng JQ. A functional variant in HOXA11-AS, a novel long non-coding RNA, inhibits the oncogenic phenotype of epithelial ovarian cancer. Oncotarget. 2015;33:34745-57.

6. Sun M, Nie F, Wang Y, Zhang Z, Hou J, He D, Xie M, Xu L, De W, Wang Z, Wang J. LncRNA HOXA11-AS promotes proliferation and invasion of gastric cancer by scaffolding the chromatin modification factors PRC2, LSD1, and DNMT1. Cancer Res. 2016;21:6299-310.

7. Wang Q, Zhang J, Liu Y, Zhang W, Zhou J, Duan R, Pu P, Kang C, Han L. A novel cell cycle-associated IncRNA, HOXA11-AS, is transcribed from the 5prime end of the HOXA transcript and is a biomarker of progression in glioma. Cancer Lett. 2016;2:251-9.

8. Z Zhang JX, Han L, Bao ZS, Wang YY, Chen LY, Yan W, Yu SZ, Pu PY, Liu N, You YP, Jiang T, Kang CS. HOTAIR, a cell cycle-associated long noncoding RNA and a strong predictor of survival, is preferentially expressed in classical and mesenchymal glioma. Neuro-Oncology. 2013;12:1595-603.

9. Zheng Q, Han L, Dong Y, Tian J, Huang W, Liu Z, Jia X, Jiang T, Zhang J, Li $X$, Kang C, Ren H. JAK2/STAT3 targeted therapy suppresses tumor invasion via disruption of the EGFRvIII/JAK2/STAT3 axis and associated focal adhesion in EGFRvIll-expressing glioblastoma. Neuro-Oncology. 2014;9:1229-43.

10. Tyagi N, Marimuthu S, Bhardwaj A, Deshmukh SK, Srivastava SK, Singh AP, McClellan S, Carter JE, Singh S. P-21 activated kinase 4 (PAK4) maintains stem cell-like phenotypes in pancreatic cancer cells through activation of STAT3 signaling. Cancer Lett. 2016;2:260-7.

11. Zhang K, Zhu S, Liu Y, Dong X, Shi Z, Zhang A, Liu C, Chen L, Wei J, Pu P, Zhang J, Jiang T, Han L, Kang C. ICAT inhibits glioblastoma cell proliferation by suppressing Wnt/ß-catenin activity. Cancer Lett. 2015;1:404-11.

12. Yin J, Park G, Kim TH, Hong JH, Kim YJ, Jin X, Kang S, Jung JE, Kim JY, Yun H, Lee JE, Kim M, Chung J, Kim H, Nakano I, Gwak HS, Yoo H, Yoo BC, Kim $J$ H, Hur EM, Lee J, Lee SH, Park MJ, Park JB. Pigment epithelium-derived factor (PEDF) expression induced by EGFRvIll promotes self-renewal and tumor progression of Glioma stem cells. PLoS Biol. 2015;5:e1002152.

13. Li L, Puliyappadamba VT, Chakraborty S, Rehman A, Vemireddy V, Saha D, Souza RF, Hatanpaa KJ, Koduru P, Burma S, Boothman DA, Habib AA. EGFR wild type antagonizes EGFRvIll-mediated activation of met in glioblastoma. Oncogene. 2015;1:129-34.

14. Ellis HP, Greenslade M, Powell B, Spiteri I, Sottoriva A, Kurian KM. Current challenges in Glioblastoma: Intratumour heterogeneity, residual disease, and models to predict disease recurrence. Front Oncol. 2015;5:251.

15. Kalkan R. Glioblastoma stem cells as a new therapeutic target for Glioblastoma. Clin Med Insights Oncol. 2015;9:95-103.

16. Kaufhold S, Garbán H, Bonavida B. Yin Yang 1 is associated with cancer stem cell transcription factors (SOX2, OCT4, BMI1) and clinical implication. J Exp Clin Cancer Res. 2016;35:84.

17. Seymour T, Nowak A, Kakulas F. Targeting aggressive cancer stem cells in Glioblastoma. Front Oncol. 2015;5:159.

\section{Submit your next manuscript to BioMed Central and we will help you at every step:}

- We accept pre-submission inquiries

- Our selector tool helps you to find the most relevant journal

- We provide round the clock customer support

- Convenient online submission

- Thorough peer review

- Inclusion in PubMed and all major indexing services

- Maximum visibility for your research

Submit your manuscript at www.biomedcentral.com/submit
) Biomed Central 